\title{
Reliability and Validity of Depression Anxiety Stress Scale (DASS)-21 in Screening Common Mental Disorders Among Postpartum Women in Malawi
}

Ernest Moya ( $\sim$ moyaernest@gmail.com )

Training and Research Unit of Excellence (TRUE)

Leila M. Larson

University of South Carolina, Arnold School of Public Health

Robert C. Stewart

University of Edinburgh, Royal Edinburgh Hospital

Jane Fisher

Monash University

Martin N. Mwangi

Training and Research Unit of Excellence (TRUE)

Kamija S. Phiri

Training and Research Unit of Excellence (TRUE)

\section{Research Article}

Keywords: Anxiety, Depression, internal reliability, receiver operating curve, sensitivity, specificity

Posted Date: February 15th, 2022

DOI: https://doi.org/10.21203/rs.3.rs-1182206/v1

License: (9) (i) This work is licensed under a Creative Commons Attribution 4.0 International License.

Read Full License 


\section{Abstract}

Background:Approximately one in five women who have recently gave birth suffer from some form of common mental disorder (CMD) particularly depression and/or anxiety.Most available CMD screening tools in most lowand middle income countries do not screen for more than one mental health problem. Having a screening tool that is free to use, short in assessment time and used to screen for more than one CMD is appealing in a resource constrained setting. This study aimed at assessing the reliability and validity of the Depression, Anxiety, and Stress Scale-21 items (DASS-21) for screeningCMDsexperienced by women who have recently given birth in Malawi.

Method: We used the gold-standard design for scale validation of comparing the responses to the Chichewa translated and adapted DASS-21 instrument with an independently administered Structured Clinical Interview for DSM-IV (SCID). Internal reliability was reported using Cronbach's alpha coefficient. The ability of DASS-21 and Edinburgh Postpartum Depression Scale (EPDS) to discriminate cases from non-cases was assessed by receiver operating characteristics (ROC) analysis and its curve was used to select cut-off points for DASS-21 and EPDS that maximise both sensitivity and specificity.

Results: The overall Cronbach's alpha for DASS-21 scale was 0.74 . The internal consistency for DASS-21 depression subscale, anxiety subscale and stress subscale were $0.66,0.29$ and 0.52 respectively. The area under the ROC curve was 0.76 (95\% Cl: 0.6086; 0.9102) for DASS-21 depression subscale (DASS-D) and 0.65 for DASS-21 anxiety subscale (DASS-A). At a cut-off point of one or more, the sensitivity and specificity for DASS-D was $69.2 \%$ and $75.5 \%$ whilst DASS-A was $52.9 \%$ and $75.5 \%$ respectively. Pearson correlation coefficient for the association between DASS-D and EPDS was $r=0.61, p<0.001$.

Conclusion: DASS-21 as an overall item has good internal reliability but none of its subscales (DASS-D, DASS-A, DASS-S) have satisfactory internal reliability.

\subsection{Background}

Maternal mental health problems remain a global public health challenge. Approximately $13 \%$ of women who have given birthin high income countries suffer from some form of common mental disorder (CMD), primarily depression and/oranxiety.[1] The situation is worse in low and lower-middle income countries (LMICs) where one in five (19.8\%) womenwho have recently given birth experience a CMD.[1] Untreated CMDs reduce maternal level of functioning,[2] and may consequently impair mother-infant interaction[3] and infant cognitive development.[4]

The current low detection and treatment rate of CMDs in LMICs is very concerning. There are several reasons for this. First, perinatal mental health for women living in LMICs is only an emerging area of interestfor researchers and policy makers, asthe international agenda has moved beyond survival to understand what is needed to enable women (and infants) to thrive.[1]Second, it has been thought that women living in LMICs are protected from experiencing postpartum mental disorders by social and traditional cultural practices during the postpartum period, a notion which has not been substantiated. 
[1]Third, low detection and treatment rate of CMDs in LMICs might be attributed to lack ofculturally sensitive screening tools that are simple to use. Lastly but important,in most LMICs perinatal mental health screening and treatmentareyet to be integrated into a routine healthcare package of mother-child dyadic interventionsoffered when a mother attends well-baby clinics.[5]

Several perinatal CMD screening tools such as Edinburgh Postnatal Depression Scale (EPDS),[6] WHO Self-Reporting Questionnaire,[7] Patient Health Questionnaire-9,[8]and Post-Traumatic Stress Scale[9]have been validated for screening perinatal mental disorders in Malawi. However, these individual tools are not designed to screen formultiple separate common mental disorders (e.g. depression, anxiety, PTSD). Having a screening tool that is free to use, short in assessment time and used to screen for more than one CMDs is more appealing in a resource constrained setting. This study aimed at assessing the reliability and validity of the Depression, Anxiety, and Stress Scale-21 items (DASS-21) for screening common mental disorders among postpartum women in Malawi. Specifically, we aimed to 1) translateinto Chichewa and adapt the DASS-21,2) in postnatal women, establish the reliability of DASS21 and its subscales and the criterion validity of the adapted DASS-21 againstthe gold standardofDiagnostic and Statistical Manual of Mental Disorders, $4^{\text {th }}$ edition (DSM-IV)diagnosis of major or minor depression and generalised anxiety disorder, and 3)compare the test characteristics ofthe DASS21 depression scale and EPDS.

\subsection{Methods}

The gold-standard design for scale validation of comparing the responses to the Chichewa translated and adapted DASS-21 instrument with a blinded independent administration of theStructured Clinical Interview for DSM-IV(SCID) and the EPDS was used. Chichewa is the widely used language in most parts of Malawi.

\subsection{Participants}

Participants were women who had recently given birth in Zomba district, southern Malawi who participated in theRandomised controlled trial of the Effect of intravenous iron on Anaemiain Malawian Pregnant women (REVAMP) trial. The original study randomized 862 anaemic (haemoglobin level between $5 \mathrm{~g} / \mathrm{dl}$ and $10 \mathrm{~g} / \mathrm{dl}$ ) pregnant women in their second trimester to either standard treatment of oral ferrous sulphate (200mg twice a day for 90 days or until birth whichever came first)or intravenous ferric carboxymaltose $(20 \mathrm{mgs} / \mathrm{kg}$ or maximum of $1000 \mathrm{mg}$ for $\geq 50 \mathrm{kgs}$ ) once for the whole duration of pregnancy (https://www.anzctr.org.au/Trial/Registration/TrialReview.aspx?id=375587). We included a sub-set of participants who came for their follow-up visits with babies aged between 1 and 12 months.

\subsection{Measures}

2.2.1 Depression Anxiety Stress Scale (DASS-21) 
The DASS-21 is the most widely used screening tool which has the capacity to separately measure symptoms of depression, anxiety, and stress. The DASS-21 has been derived from the original 42-items DASS developed by Lovibond et.al (1995) which has three sub-scales namely Depression subscale (DASS-D), Anxiety subscale (DASS-A) and Stress subscale (DASS-S).[10]The DASS-D measures individual's hopelessness, positive affect and self-esteem. The DASS-A measures autonomic arousal, situational anxiety, musculo-skeletal symptoms, perceived experience of anxiety arousal and situation anxiety. Finally, DASS-S measures agitation, tension and negative affect.[11]Each subscale comprises of 7 items that are scored from 0 (did not apply to me at all) to 3 (applied to me very much, or most of the time) for the week preceding the interview to reflect severity. The total score for each DASS-21 subscale ranges from 0 to 21.The items scores are added and thereafter multiplied by two to obtain the total score that can be compared with the original DASS-42. The DASS-21 has been translated into more than 54 languages (http://www2.psy.unsw.edu.au/groups/dass/translations.htm) but not in Chichewa and has demonstrated good internal consistency and validity. However, very few studies have established cut-off points for severity classification hence limiting its use in clinical settings.[12]

\subsubsection{Edinburgh Postnatal Depression Scale (EPDS)}

The EPDS is a ten-item self-report scale that has been widely used to detect clinically significant depressive symptomsexperienced by women during the postnatal period.Each question is rated on a scale of 0 to 3 , and total scores can range from 0 to 30, with a higher score representing more depressive symptoms. If criteria for culturally sensitive translation are met, EPDS has shown to be a reliable screening tool for depression in LMICs.[13]Some authors suggest that EPDS itemsnumber 3, 4 and 5(EPDS-3A) that assess guilt, anxiety and fear can detect anxiety disorders. However,studies have reported mixed findings on the reliability of EPDS-3A in screening symptoms of anxiety in other settings. [14-17] In Malawi, the EPDS has been validated for useamong women who were pregnant, and the Chichewa-translatedEPDS has shown to be a valid tool for screening and detecting episodes of both major and minor depression among antenatal women (AUC=0.767: 95\% Cl 0.695-0.839).[6]

\subsubsection{Diagnostic and Statistical Manual of Mental Disorders: Fourth Edition (DSM-IV)}

The DSM-IV remains the gold standard tool for making diagnosis of mental disorders. The Structured Clinical Interview for DSM-IV (SCID) uses categorical classifications to divide mental disorders based on set of criteria with defining features. It requires an individual with clinical training and experience to administer the SCID. The specific diagnostic criteria that are included in the SCID are only meant to guide but a diagnosis is informed by clinical judgment. This study used SCID modules for depression (minor and major) and anxiety (current generalised anxiety disorder) previously translated into Chichewa, adapted and used in Malawi.[18]

\subsection{Procedures}

\subsubsection{Content Validation}


The DASS-21 was translated from English into Chichewa (Appendix 1) by professional accredited translators at Malawi Liverpool Wellcome Trust Research Centre in Malawi. Thereafter, it was reviewed by a group of health workers and mental health clinicians for appropriateness of language and cultural idioms. Through this meeting, it was agreed that DASS-Stress subscale item questions measure different constructs from those in the stress module in DSM-V,and assessment for criterion validation hastherefore not be done for this subscale.A field trial of the reviewed DASS-21 was conducted with20 women who were pregnant and contributedinformation on clarity, comprehensibility, and suggestions of culturally sensitive phrases. The tool was then back-translated by an independent professional translator for verification.

\subsubsection{Construct and Criterion Validation}

\subsubsection{Sample Size}

Assumingthat the prevalence of postpartum depression wasat $10 \%,[6,19]$ a minimum sample of 120 participants (including 12 having clinical symptoms of depression) was adequate to achieve a minimum power of $90 \%$ to detect a change in the percentage value of sensitivity of DASS-21 items from 0.50 to 0.90 based on the target significance level of 0.05 . This sample size wasalso adequate to detect a change in value of specificity from $60 \%$ to $80 \%$ which only required a minimum of 50 participants (including five participants with clinical signs of depression). The sample size was calculated based on prevalence of depression as it is the most common andsignificant public health with serious clinical impactcompared to anxiety.[20]

\subsubsection{Questionnaire administration}

We used a convenience sampling technique to select 120 participants from the REVAMP trial participants who came for their scheduled visits with babies aged between one and 12 months. A maximum of five consecutive participants were assessed in a day to ensure quality assessments.Data collection was performed between November 2020 and May 2021. Participants were first interviewed by 1) a mental health clinician (TK) who administered the SCID modules for major or minor depression, current and generalised anxiety disorder and 2) a trained registered nurse (GK) administered the DASS-21 and EPDS in individual interviewers thereafter. This is usual and best practice in settings where there is low familiarity with test-taking and where many people have low literacy. The two assessors workedindependently, and were blinded to each other's scores.

\subsection{Data Management and Quality Assurance}

Thepaper based SCID administered by themental health clinician waschecked for completeness by the Corresponding author (EM) before entry in Open Data Kit (ODK). The DASS-21 and EPDS were directly collected using a tablet (ODK) and data were checked for completeness before being uploaded to the server. Data were cleaned and checked for consistency before analysis by EM.

\subsection{Statistical Analysis}

Page 5/21 


\subsubsection{Internal reliability}

The reliability of any given measurement is the extent to which it is a consistent measure of a concept. Internal reliability was measured by Cronbach's alpha which reflects the extent to which different subsets of the test items produce similar measures.[21] We have reported both overall alpha and instrument (DASS-21) subscale alphas. The alpha coefficient of reliability ranges from 0 (all items are entirely independent from one another) to 1 (all items are highly related). Alpha coefficient between 0.70 and 0.80 will be regarded as acceptable, and less than 0.5 as not acceptable.[22]Further analysis of the internal consistency reliability was done by calculating item-test, item-rest and average inter-item correlations. All these influencesand describes the overall item score reliability.

\subsubsection{Correlation between DASS-21 subscale of depression and EPDS}

We examined convergent validity of the DASS- Depression subscale by assessing its correlation with EPDS, a scale that has demonstrated to be a valid depression screening measure in Malawi.A Pearson's correlation coefficient ( $r$ ) for DASS-21 subscale of depression and EPDS was also calculated as the scores in both tools were normally distributed. The $r$ correlation measures the strength of linear relationship between two continuous variables. The following cut-off $0.1<[r]<0.3,0.3<[r]<0.5$ and 0.5 $<[r]<0.7$ and $[r]>0.7$ were used to measure the strength of association and interpreted as very weak correlation, weak correlation, moderate correlation and strong correlation respectively.[24]

\subsubsection{Criterion Validation for DASS-D, DASS-A and EPDS against the SCID}

Compared tothe scores from the DSM-IV structured interviews (SCID), we calculated the sensitivity, specificity, positive and negative likelihoodratio for DASS-21 and EPDS.Positive likelihood ratio (LR+) tells us how much to increase the probability of the disease if the test is positive while Negative likelihood ratio (LR-) tells us how much to decrease the probability of the disease if the test is negative. The larger the LR+ $(\geq 5)$, the greater the likelihood of the disease and the smaller the LR- $(<5)$, the lesser the likelihood of a disease. (http://medtrain.chm.msu.edu/ebm/Diagnosis/at_likelihood_ratios.html).

The ability of DASS-21 and EPDS to discriminate cases from non-cases was assessed by receiver operating characteristics (ROC) analysis and its curve was used to select cut-off points for DASS-21 and EPDS. The area under the ROC curve (AUC) is the best parameter for summarizinga screening tool's overall discriminative value. A value of $\geq 0.70,0.80$ up to 0.90 and $>0.90$ is interpreted as reasonable, good and excellent, respectively.[23]Comparisons were made between the AUCs for the DASS-D and EPDS. The criteria to choose an optimal cut-off value was to maximise both sensitivity and specificity, and to set sensitivity value higher than specificity in order to detect all potential cases. For a test to be useful, the sensitivity + specificity should be atleast 1.5.Data analysis was conducted using Stata Version 15.1 (StataCorp LP, College Station, Texas, United States of America, 2017).

\subsection{Ethical Considerations}


This study was nested within the REVAMP trial, which was approved by the College of Medicine Research and Ethics Committee, Malawi (P.02/18/2357) and the Walter and Eliza Hall Institute of Medical Research Ethics Committee,Australia(WEHI REC 18/02). All participants were given information about the study and either signed a consent form or printed their thumbprint (as witnessed by impartial observer) for those who could not write. Assessments were done in closed rooms to maintain privacy. Participants were given unique identifying numbers and forms containing their names were kept separately from their files. Participants identified as needing further assessment and management were referred to local Ministry of Health psychiatric services.

\subsection{Results}

\section{1 Descriptive analysis}

Overall, $115 / 120$ (95.8\%) of the required sample provided complete responses to all the three questionnaires (SCID, DASS-21 and EPDS) administered. The mean age of the included participants was 24 (standard deviation: 6.8) years. Approximately $82 \%$ of the participants were either married or living together with their husband and $68.7 \%$ of them were Christians (Table 1). Most of the participants had either attended primary $(60.9 \%)$ or secondary $(34.8 \%)$ school and depended on either casual labour $(35.7 \%)$ or farming $(36.5 \%)$ to earn a living.

Using the SCID interviews, 13/115 (11.3\%) participants were diagnosed with current depression (3 with major depression and 10 with minor depression) and 17/115 (14.8\%) were diagnosed with current generalised anxiety disorder. The 3 participants diagnosed with major depression were referred to local psychiatric services for further management.

\subsection{Internal reliability of DASS-21 items and EPDS}

The results of the overall DASS-21 item test scale reliability are shown in Table 2 . The overall Cronbach's alphafor DASS-21 was 0.74 . The Cronbach's alpha for DASS-D subscale, DASS-A subscale and DASS-S subscale were $0.66,0.29$ and 0.52 respectively. Detailed results on item-test correlation, item-rest correlation and average inter-item correlation which influences the overall Cronbach's alpha are shown in Table 2. The internal reliability (Cronbach's alpha) of EPDS was 0.74 with item-test correlation and item specific alpha ranging from 0.30 to 0.81 and 0.67 to 0.76 respectively.

\subsection{Correlation between DASS-D subscale and EPDS}

A Pearson correlation coefficient examined the relationship between scores on the DASS-D subscale and EPDS. The results showed a statistically significant positive and moderate association $(r=0.61$, $p<0.001)$.

3.4Criterion Validityfor DASS-21, DASS-D, DASS-A and EPDS against SCID 
The results of sensitivity, specificity, cases correctly classified, positive likelihood ratio (LR+) and negative likelihood ratio (LR-) for both DASS-21 (Depression and Anxiety sub-scales) and EPDS compared to SCID are shown in Table 3.The receiver operating curve (ROC) analysis for DASS-21 depression subscale against criterion of DSM-IV current depressive episode (minor or major) gave an area under the curve (AUC) value of 0.7594 (95\% Cl: 0.6086; 0.9102)and details are shown in Figure 1A.The ROC analysis for DASS-21 anxiety subscale against criterion of DSM-IV current generalized anxiety disorder gave AUC value of 0.6505 (95\% Cl: 0.5150; 0.7857) as shown in Figure 1B.The ROC analysisfor EPDS against criterion of DSM-IV current depressive episode (minor or major) gave AUC value of $0.7485(95 \% \mathrm{Cl}$ : $0.6031 ; 0.8939)$. As shown in Figure $1 \mathrm{~A}$, although the AUC for DASS-depression subscale is larger than that of EPDS, the chi-squared test yielded a probability of 0.8844 , suggesting that there is no significance difference between the two areas. The ROC area for the whole DASS-21 against the SCID depression and anxiety were 0.7059 (95\% Cl: $0.5433 ; 0.8685)$ and 0.7695 (95\% Cl: $0.6228 ; 0.9162)$ respectively.

\subsection{Choice of cut-off points}

A cut-off point of 1 or higher on the DASS-D depression subscale provided the best combination of sensitivity and specificity (optimal point) in detecting a diagnosis of either minor or major depression. This cut-off point gave $69.2 \%$ and $75.5 \%$ for sensitivity and specificity respectively. At this cut-off point ( 1 or higher), the correct classification was $74.8 \%$ with positive likelihood ratio of 2.8 and negative likelihood ratio of 0.4 (Table 3).For the DASS-A (anxiety subscale), the optimal cut-off point was obtained at a cutoff point of 1 or higher that gave a sensitivity of $52.9 \%$ and specificity of $75.5 \%$ (Table 3 ). This cut-off point gave a correct classificationvalue of $72.2 \%$, and 2.16 and 0.62 for positive and negative likelihood ratio respectively (Table 3 ).For EPDS, the best combination for sensitivity and specificity was $76.9 \%$ and $66.7 \%$ respectively at a cut-off point of 1 or higher. This cut-off point gave correct classification value of $72.2 \%$ with positive likelihood ratio value of 2.16 and negative likelihood ratio value of 0.62 (Table 3).

\subsection{Discussion}

\subsection{Reliability and Validity of DASS-21}

The aim of this study was to determine the reliability and validity of DASS-21 in screening for common mental disorders (CMDs) among postpartum women in Malawi. The findings indicated that the internal consistency of the whole DASS-21 was good (Cronbach's alpha=0.74). However, internal consistency wasunsatisfactory for DASS-21 depression subscale and stress subscale, and poor for DASS-21 anxiety subscales. Our findings are not consistent with what has been reported in high income countries where the Cronbach's alpha coefficient for the DASS-21 subscales ranged from good to excellent indicating good internal consistency.[25-27] A study in Nigeria also reported high Cronbach's alpha for all DASS-21 subscales.[28] These discrepancies in findings might be due to difference in participants' level of education among studies. Most of the participants in our study had received primary school education compared to a study in Nigeria which involved medical students and high-income countries where majority of the study participants had a minimum of first degree. It has been suggested that participants 
with low literacy might have difficulties in understanding and rating subjective emotional state presented in a Likert scale.[26] Stewart et. al (2009) addressed the challenges of Likert scale ratingamong low literate participants by introducing pictorial faces that demonstrates variation in emotional state.[6] However, these additions might add complexity in tool use which will require adequate training before use and also raises feasibility constraints especially in LMICs where resources are limited.

It is known that DASS-21 anxiety subscale items measure two different constructs namely somatic symptoms and negative emotional symptoms. Additionally, it has been suggested that women with common mental disorders tend to endorse more somatic symptoms than negative emotions.[29]This was evident in this study as three of DASS-21 anxiety subscale items indicated negative inter-item correlation (Table 2). This consequently reduced the average inter-item correlation as items that are not related and going in different directions end up cancelling each other thereby affecting the overall Cronbach alpha (Cronbach alpha coefficient for DASS-A subscale $=0.29$ ).

Another important finding in this study is that none of the DASS-21 item subscales combined sufficiently to give high sensitivity and specificity for routine screening for common mental disorders even at a cutoff point of 1 or more. Similar findings were reported by Tran et. al (2013) in the validation of the DASS21 as a screening instrument for depression and anxiety in a rural community-based cohort of northern Vietnamese women.Using exploratory factor analysis, Tran et. al (2013)found DASS-21 being not good at distinguishing different conditions, but the single factor in which all subscales are added had some value in detecting any CMD.[29] Hanlon et al (2015) argued that in low-literacy settings, it is possible that potential accuracy of a screening tool can be off-set by the complexity of the multiple response categories presented in Likert scales.[30] The area under the ROC curve for DASS-21 depression subscale was found to be 0.76 and EPDS was 0.75 , this is moderately high value suggesting reasonable diagnostic ability of both DASS depression subscale and EPDS. The AUC for EPDS in this study is almost similar to what was reported (0.77: $95 \% \mathrm{Cl} ; 0.695-0.839)$ in a previous study that validated use of EPDS in screening minor or major depression among antenatal women in our setting.[6]Comparison of AUCs between EPDS (which is an established tool in our setting for screening postpartum depression) and DASS-D subscale indicated no significance difference between the two areas. Our results have also showed a statistically significant positive and moderate association between DASS- 21 depression subscale and EPDS score suggesting DASS-21 depression subscale as a reliable tool for screening minor or major depression.

\subsection{Strength and Limitation}

This study is the first study in Malawi to use the criterion validation (gold standard) against DASS-21 subscales which is the most acceptable method in the field of perinatal health. We used Cronbach's alpha which is a standard analysis of internal reliability (item inter-relatedness) of psychometric tools. However, we acknowledged that we did not do test-retest reliability due to resource constraints. Our study findings may have reduced generalisability as we only included mothers who had babies aged between one and 12 months old who were previously enrolled in a randomisation study which recruited only anaemic 
pregnant women. Lastly, most of the participants in this study received primary education and the findings should not be generalised in a population with high literacy level.

\subsection{Conclusion}

In summary, the study found DASS-21 as an overall item having good internal reliability but none of its subscales (DASS-A and DASS-S) had satisfactory internal reliability except for the depression subscale. There was a positive andstatistically significant moderate correlation between DASS-D subscale and the EPDS and that there is no significance difference between their ability to detect either minor or moderate depression among women who have recently given birth.Although the DASS-21 depression subscale can be used with caution in screening for minor or major depression in this setting, it is not recommended to use the other subscales independently due to their low internal reliability consistency, sensitivity and specificity values.

\section{Abbreviations}

CMDs: Common Mental Disorders; DASS-21: Depression Anxiety Stress Scale-21 items; EPDS: Edinburgh Postpartum Depression Scale; GK: Gladys Kusiwa; LMICs: Lower and Middle-Income Countries; ROC: Receiver Operating Characteristics; SCID: Structured Clinical Interview for DSM-IV; TK: Twisile Kalinga

\section{Declarations}

\section{Ethics approval and informed consent}

The protocol was approved by College of Medicine Research and Ethics Committee, Malawi (P.02/18/2357) and the Walter and Eliza Hall Institute of Medical Research Ethics Committee, Australia (WEHI REC 18/02) in accordance with the International Conference on Harmonisation Guidelines for good clinical practice and the Declaration of Helsinki.

\section{Consent for Publication}

Not applicable

\section{Availability of data and material}

The datasets generated and/or analysed during the current study are not publicly available as the main study (REVAMP trial) is still ongoing but are available from the corresponding author on reasonable request.

\section{Competing Interests}

All authors declare that they have no competing interests 


\section{Funding}

This manuscript is not directly funded. However, the corresponding author is PhD student supported by CARTA. CARTA is jointly led by the African Population and Health Research Center and the University of the Witwatersrand, South Africa and is funded by Sida (Grant No: 54100113), Carnegie Corporation of New York (Grant No. G-19-57145), the DELTAS Africa Initiative (Grant No: 107768/Z/15/Z). The DELTAS Africa Initiative is an independent funding scheme of the African Academy of Sciences (AAS)'s Alliance for Accelerating Excellence in Science in Africa (AESA) and supported by the New Partnership for Africa's Development Planning and Coordinating Agency (NEPAD Agency) with funding from the Wellcome Trust (UK) and the UK government. CARTA did not play any role in the design, collection, analysis and interpretation of data.

\section{Authors' Contributions}

$\mathrm{EM}, \mathrm{LML}, \mathrm{JF}, \mathrm{MM}$ and $\mathrm{KP}$ were involved in conception of research work and data collection. RS was involved in the design and statistical analysis. EM prepared figures, analysed the data and drafted the manuscript. All authors read and approved the final version of the manuscript

\section{Acknowledgement}

I would like to express my sincere gratitude to the Consortium of Advanced Research and Training in Africa (CARTA) JAS-3 Facilitators for their untiring support during writing workshop. I would also like to extend my gratitude to Gladys Kusiwa (GK) and Twisile Kalinga (TK) for their contribution during data collection period. Lastly special gratitude to all clients who participated in this study.

\section{References}

1. Fisher J, Mello C De, Patel V, Rahman A, Tran T, Holmes W (2012) Prevalence and determinants of common perinatal mental disorders in women in low- and lower-middle-income countries: a systematic review.Bull World Health Organ $h t t p: / / w w w . d o i: 10.2471 / B L T .11 .091850$

2. Barkin JL, Wisner KL, Bromberger JT, Beach SR, Wisniewski SR (2016) Factors Associated with Postpartum Maternal Functioning. 25:707-713.Journal of Women's Health http://www.doi:10.1089/jwh.2015.5296

3. Lehnig F, Nagl M, Stepan H, Wagner B, Kersting A (2019) Associations of postpartum mother-infant bonding with maternal childhood maltreatment and postpartum mental health: a cross-sectional study. BMC Pregnancy and Childbirth.https://doi.org/10.1186/s12884-019-2426-0

4. Slomian J, Honvo G, Emonts P, Reginster JY, Bruyère $O$ (2019) Consequences of maternal postpartum depression: A systematic review of maternal and infant outcomes. Women's Heal. http://doi: 10.1177/1745506519844044

5. Baron EC, Hanlon C, Mall S, et al (2016) Maternal mental health in primary care in five low- and middle-income countries: a situational analysis. BMC Health Serv Res. http://doi: 10.1186/s12913- 
016-1291-z

6. Stewart RC, Umar E, Tomenson B, Creed F (2013) Validation of screening tools for antenatal depression in Malawi - A comparison of the Edinburgh Postnatal Depression Scale and Self Reporting Questionnaire. J Affect Disord 150:10411047http://dx.doi.org/10.1016/j.jad.2013.05.036

7. Stewart RC, Kauye F, Umar E, Vokhiwa M, Bunn J, Fitzgerald M, Tomenson B, Rahman A, Creed F (2009) Validation of a Chichewa version of the Self-Reporting Questionnaire (SRQ) as a brief screening measure for maternal depressive disorder in Malawi, Africa. J Affect Disord 112:126134.http://dx.doi.org/10.1016/j.jad.2008.04.001

8. Udedi M, Muula AS, Stewart RC, Pence BW (2019) The validity of the patient health Questionnaire-9 to screen for depression in patients with type-2 diabetes mellitus in non-communicable diseases clinics in Malawi. BMC Psychiatry 19:1-7 https://doi.org/10.1186/s12888-019-2062-2

9. Gondwe Whitney K, Yang Q, Brandon D, Chirwa E, Holditch-davis D (2018) International Journal of Africa Nursing Sciences Validation of the Chichewa Perinatal PTSD Questionnaire and Chichewa Child Health Worry Scale. Int J Africa Nurs Sci 9:42-48https://doi.org/10.1016/j.ijans.2018.07.003

10. Moussa MT, Lovibond P, Laube R, Megahead HA (2017) Psychometric Properties of an Arabic Version of the Depression Anxiety Stress Scales (DASS). Res Soc Work Pract 27:375386http:10.1177/1049731516662916

11. Tran TD, Tran T, Fisher J (2013) Validation of the depression anxiety stress scales ( DASS ) 21 as a screening instrument for depression and anxiety in a rural community-based cohort of northern Vietnamese women. BMC Psychiatry http://www.biomedcentral.com/1471-244X/13/24

12. Lee J, Lee EH, Moon SH (2019) Systematic review of the measurement properties of the Depression Anxiety Stress Scales-21 by applying updated COSMIN methodology. Qual Life Res 28:23252339https://doi.org/10.1007/s11136-019-02177-x

13. Shrestha SD, Pradhan R, Tran TD, Gualano RC, Fisher JRW (2016) Reliability and validity of the Edinburgh Postnatal Depression Scale ( EPDS ) for detecting perinatal common mental disorders ( PCMDs ) among women in low-and lower-middle-income countries: a systematic review. BMC Pregnancy Childbirth. http: doi: 10.1186/s12884-016-0859-2

14. Matthey S, Fisher J, Rowe H (2013) Using the Edinburgh postnatal depression scale to screen for anxiety disorders: Conceptual and methodological considerations. J Affect Disord 146:224230http://dx.doi.org/10.1016/j.jad.2012.09.009

15. Smith-Nielsen J, Egmose I, Wendelboe KI, Steinmejer P, Lange T, Vaever MS (2021) Can the Edinburgh Postnatal Depression Scale-3A be used to screen for anxiety? BMC Psychol 9:1-11. https://doi.org/10.1186/s40359-021-00623-5

16. Simpson W, Glazer M, Michalski N, Steiner M, Frey BN (2014) Comparative efficacy of the generalized anxiety disorder 7-item scale and the Edinburgh Postnatal Depression Scale as screening tools for generalized anxiety disorder in pregnancy and the postpartum period. Can J Psychiatry 59:434-440. http://10.1177/070674371405900806

Page $12 / 21$ 
17. Van Der Zee-Van den Berg Al, Boere-Boonekamp MM, Groothuis-Oudshoorn CGM, Reijneveld SA (2019) The Edinburgh Postpartum Depression Scale: Stable structure but subscale of limited value to detect anxiety. PLoS One 14:1-10https://doi.org/10.1371/journal. pone.0221894

18. Mittal VA, Walker EF (2011) Diagnostic and Statistical Manual of Mental Disorders. Psychiatry Res. http://doi: 10.1016/j.psychres.2011.06.006

19. Anna Dow, Queen Dube, Brian W. Pence and AVR (2014) Postpartum Depression and HIV Infection among Women in Malawi. J Acquir Immune Defic Syndr 65:359365https://www.ncbi.n/m.nih.gov/pmc/articles/PMC3624763/pdf/nihms412728.pdf

20. Bujang MA, Adnan TH (2016) Requirements for Minimum Sample Size for Sensitivity and Specificity Analysis. http://doi: 10.7860/JCDR/2016/18129.8744

21. Tang W, Cui Y, Babenko O (2014) Internal consistency: Do we really know what it is and how to assess it? Internal Consistency https://www.researchgate.net/publication/280839401

22. Taber KS (2018) The Use of Cronbach 's Alpha When Developing and Reporting Research Instruments in Science Education. 1273-1296http:// doi:10.1007/s11165-016-9602-2

23. Mandrekar JN (2010) Receiver Operating Characteristic Curve in Diagnostic Test Assessment. J Thorac Oncol 5:1315-1316. http://dx.doi.org/10.1097/JTO.0b013e3181ec173d

24. Mindrila D, Ph D, Balentyne P, Ed M (2013) Scatterplots and Correlation.

25. Minh Thi Hong Le, Thach Duc Tran, Sara Holton, Huong Thanh Nguyen, Rory Wolfe JF (2020) Reliability, convergent validity and factor structure of the DASS-21 in a sample of Vietnamese adolescents. J Affect Disord

277:110456https://www.ncbi.n/m.nih.gov/pmc/articles/PMC5516980/pdf/pone.0180557.pdf

26. Vignola RCB, Tucci AM (2014) Adaptation and validation of the depression, anxiety and stress scale (DASS) to Brazilian Portuguese. J Affect Disord 155:104109http://dx.doi.org/10.1016/j.jad.2013.10.031

27. Oei TPS, Sawang S, Goh YW, Mukhtar F (2013) Using the Depression Anxiety Stress Scale 21 (DASS21) across cultures. Int J Psychol 48:1018-1029 http://dx.doi.org/10.1080/00207594.2012.755535

28. Coker AO, Coker OO, Sanni D (2018) Psychometric properties of the 21-item Depression Anxiety Stress Scale (DASS-21). African Res Rev 12:135http://doi:10.4314/afrrev.v12i2.13

29. Tran TD, Tran T, Fisher J (2013) Validation of the depression anxiety stress scales (DASS) 21 as a screening instrument for depression and anxiety in a rural community-based cohort of northern Vietnamese women. BMC Psychiatry 13:1 http://www.biomedcentral.com/1471-244X/13/24

30. Hanlon C, Medhin G, Selamu M, Breuer E, Worku B, Hailemariam M, Lund C, Prince M, Fekadu A (2015) Validity of brief screening questionnaires to detect depression in primary care in Ethiopia. $J$ Affect Disord 186:32-39http://dx.doi.org/10.1016/j.jad.2015.07.015

\section{Tables}


Table 1: Characteristics of the study participants $(\mathrm{N}=115)$

\begin{tabular}{|c|c|}
\hline Age (in years) * & $\mathrm{n}(\%)$ \\
\hline$<20$ & $37(32.2)$ \\
\hline $20-29$ & $55(47.8)$ \\
\hline $30-39$ & 19 (16.5) \\
\hline$\geq 40$ & $4(3.5)$ \\
\hline \multicolumn{2}{|l|}{ Marital status } \\
\hline Never married & 19 (16.6) \\
\hline Married/Living together & $94(81.7)$ \\
\hline Divorced/Separated & $2(1.7)$ \\
\hline \multicolumn{2}{|l|}{ Education level } \\
\hline Not attended school & $3(2.6)$ \\
\hline Primary & $70(60.9)$ \\
\hline Secondary & $40(34.8)$ \\
\hline Tertiary & $2(1.7)$ \\
\hline \multicolumn{2}{|l|}{ Religion } \\
\hline Christianity & $79(68.7)$ \\
\hline Islam & $36(31.3)$ \\
\hline \multicolumn{2}{|l|}{ Source of income } \\
\hline Casual work & $41(35.7)$ \\
\hline Farming & $42(36.5)$ \\
\hline Permanent Employment & $32(27.8)$ \\
\hline \multicolumn{2}{|l|}{ Tribe } \\
\hline Chewa & $16(13.9)$ \\
\hline Yao & $43(37.4)$ \\
\hline Lomwe & $33(28.7)$ \\
\hline Mang'anja & $5(4.3)$ \\
\hline Tonga & $14(12.2)$ \\
\hline Others & $4(3.5)$ \\
\hline
\end{tabular}

Age* mean: 24, standard deviation (SD): 6.8 
Page 15/21 
Table 2: DASS-21 Item test scale reliability

Item question

Obs. Sign

Item-test Item-rest

correlation

Average

inter-item

Alpha

correlation

DASS-Depression subscale

I found it difficult to work up the initiative to do things

I couldn't seem to experience any positive feeling at all

I felt that I had nothing to look forward to

I felt down-hearted and blue

I was unable to become

enthusiastic about anything

I felt I wasn't worth much as a person

$115+$

0.5908

0.3907

0.2151

0.6219

$115+0.4552$

0.2263

0.2515

0.6685

$115+$

0.6124

0.4183

0.2093

0.6137

$115+$

0.5742

0.3697

0.2196

0.6280

$115+$

0.4266

0.1934

0.2592

0.6774

I felt that life was meaningless

$115+$

0.6536

0.4720

0.1983

0.5974

Test Scale Reliability (Cronbach's alpha)

0.2193

0.6629

DASS-Anxiety subscale

I was aware of dryness of my mouth

$115+0.4242$

0.1068

0.0587

0.2722

texpere

I experienced breathing difficulty

$115+\quad 0.4748$

0.1657

0.0484

0.2336

I experienced trembling (e.g. in the hands)

$115 \quad-\quad 0.3392$

0.0130

0.0760

0.3305

I was worried about situations in

$115+0.4485$

0.1347

0.0537

0.2541

which I might panic and make a

fool of myself

I felt I was close to panic

115

0.4349

0.1190

0.0565

0.2643

I was aware of the action of my heart in the absence of physical

$115+$

0.5410

0.2467

0.0349

0.1781

exertion

I felt scared without any good reason

$115 \quad-\quad 0.3961$

0.0751

0.0644

0.2923

Test Scale Reliability (Cronbach's alpha)

0.0561

0.2937

DASS-Stress subscale 


\begin{tabular}{|c|c|c|c|c|c|c|}
\hline I found it hard to wind down & 115 & + & 0.6744 & 0.4705 & 0.0963 & 0.3899 \\
\hline $\begin{array}{l}\text { I tended to over-react to } \\
\text { situations }\end{array}$ & 115 & + & 0.2857 & 0.0052 & 0.1886 & 0.5824 \\
\hline $\begin{array}{l}\text { I felt that I was using a lot of } \\
\text { nervous energy }\end{array}$ & 115 & + & 0.3669 & 0.0923 & 0.1693 & 0.5501 \\
\hline I found myself getting agitated & 115 & + & 0.6929 & 0.4963 & 0.0919 & 0.3777 \\
\hline I found it difficult to relax & 115 & + & 0.5571 & 0.3158 & 0.1241 & 0.4595 \\
\hline $\begin{array}{l}\text { I was intolerant of anything that } \\
\text { kept me from getting on with } \\
\text { what I was doing }\end{array}$ & 115 & + & 0.3083 & 0.0291 & 0.1832 & 0.5737 \\
\hline I felt that I was rather touchy & 115 & + & 0.6929 & 0.4963 & 0.0919 & 0.3777 \\
\hline $\begin{array}{l}\text { Test Scale Reliability (Cronbach's } \\
\text { alpha) }\end{array}$ & & & & & 0.1355 & 0.5233 \\
\hline
\end{tabular}


Table 3: Operating characteristics of DASS-D, DASS-A and EPDS at various cut-off scores for identifying depression and anxiety against the SCID

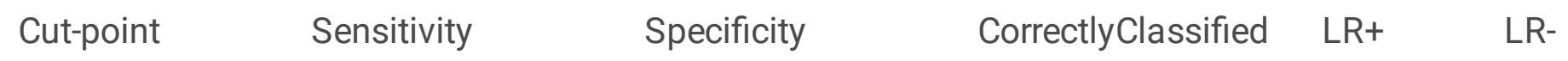

DASS-D (Depression subscale)

\begin{tabular}{llllll}
\hline$>=0$ & $100.00 \%$ & $0.00 \%$ & $11.30 \%$ & \multicolumn{2}{l}{1.0000} \\
\hline$>=1$ & $69.23 \%$ & $75.49 \%$ & $74.78 \%$ & 2.8246 & 0.4076 \\
\hline$>=2$ & $53.85 \%$ & $89.22 \%$ & $85.22 \%$ & 4.9930 & 0.5173 \\
\hline$>=3$ & $38.46 \%$ & $95.10 \%$ & $88.70 \%$ & 7.8462 & 0.6471 \\
\hline$>=4$ & $30.77 \%$ & $96.08 \%$ & $88.70 \%$ & 7.8462 & 0.7206 \\
\hline$>=6$ & $7.69 \%$ & $99.02 \%$ & $88.7 \S 0 \%$ & 7.8462 & 0.9322 \\
\hline$>=7$ & $0.00 \%$ & $99.02 \%$ & $87.83 \%$ & 0.0000 & 1.0099 \\
\hline$>=8$ & $0.00 \%$ & $100.00 \%$ & $88.70 \%$ & & 1.0000 \\
\hline & & DASS-A (Anxiety subscale) & & \\
\hline$>=0$ & $100.00 \%$ & $0.00 \%$ & $14.78 \%$ & 1.0000 & \\
\hline$>=1$ & $52.94 \%$ & $75.51 \%$ & $72.17 \%$ & 2.1618 & 0.6232 \\
\hline$>=2$ & $23.53 \%$ & $89.80 \%$ & $80.00 \%$ & 2.3059 & 0.8516 \\
\hline$>=3$ & $17.65 \%$ & $96.94 \%$ & $85.22 \%$ & 5.7647 & 0.8495 \\
\hline$>=5$ & $5.88 \%$ & $100.00 \%$ & $86.09 \%$ & & 0.9412 \\
\hline$>=6$ & $0.00 \%$ & $100.00 \%$ & $85.22 \%$ & & 1.0000
\end{tabular}

Edinburgh Postpartum Depression Scale (EPDS)

\begin{tabular}{llllll}
$>=$ & $100.00 \%$ & $0.00 \%$ & $11.30 \%$ & \multicolumn{2}{c}{1.0000} \\
\hline$>=1$ & $66.67 \%$ & $67.83 \%$ & 2.3077 & 0.3462 \\
\hline$>=2$ & $76.92 \%$ & $81.37 \%$ & $78.26 \%$ & 2.8907 & 0.5672 \\
\hline$>=3$ & $53.85 \%$ & $87.25 \%$ & $80.87 \%$ & 2.4142 & 0.7934 \\
\hline$>=4$ & $30.77 \%$ & $94.12 \%$ & $86.96 \%$ & 5.2308 & 0.7356 \\
\hline$>=5$ & $30.77 \%$ & $95.10 \%$ & $87.83 \%$ & 6.2769 & 0.7280 \\
\hline$>=6$ & $30.77 \%$ & $97.06 \%$ & $89.57 \%$ & 10.4615 & 0.7133 \\
\hline$>=7$ & $30.77 \%$ & $98.04 \%$ & $90.43 \%$ & 15.6923 & 0.7062 \\
\hline$>=8$ & $30.77 \%$ & $99.02 \%$ & $90.43 \%$ & 23.5385 & 0.7768 \\
\hline$>=9$ & $23.08 \%$ & $100.00 \%$ & $90.43 \%$ & & 0.8462
\end{tabular}




$\begin{array}{lcclc}>=12 & 7.69 \% & 100.00 \% & 89.57 \% & 0.9231 \\ >=13 & 0.00 \% & 100.00 \% & 88.70 \% & 1.0000 \\ \text { LR+: Likelihood Ratio Positive } & & \text { LR-: Likelihood Ratio Negative } & \\ \text { SCID: Structured Clinical Interviews for Diagnostic } & & \\ \text { Statistical Manual } & & & \end{array}$

\section{Figures}




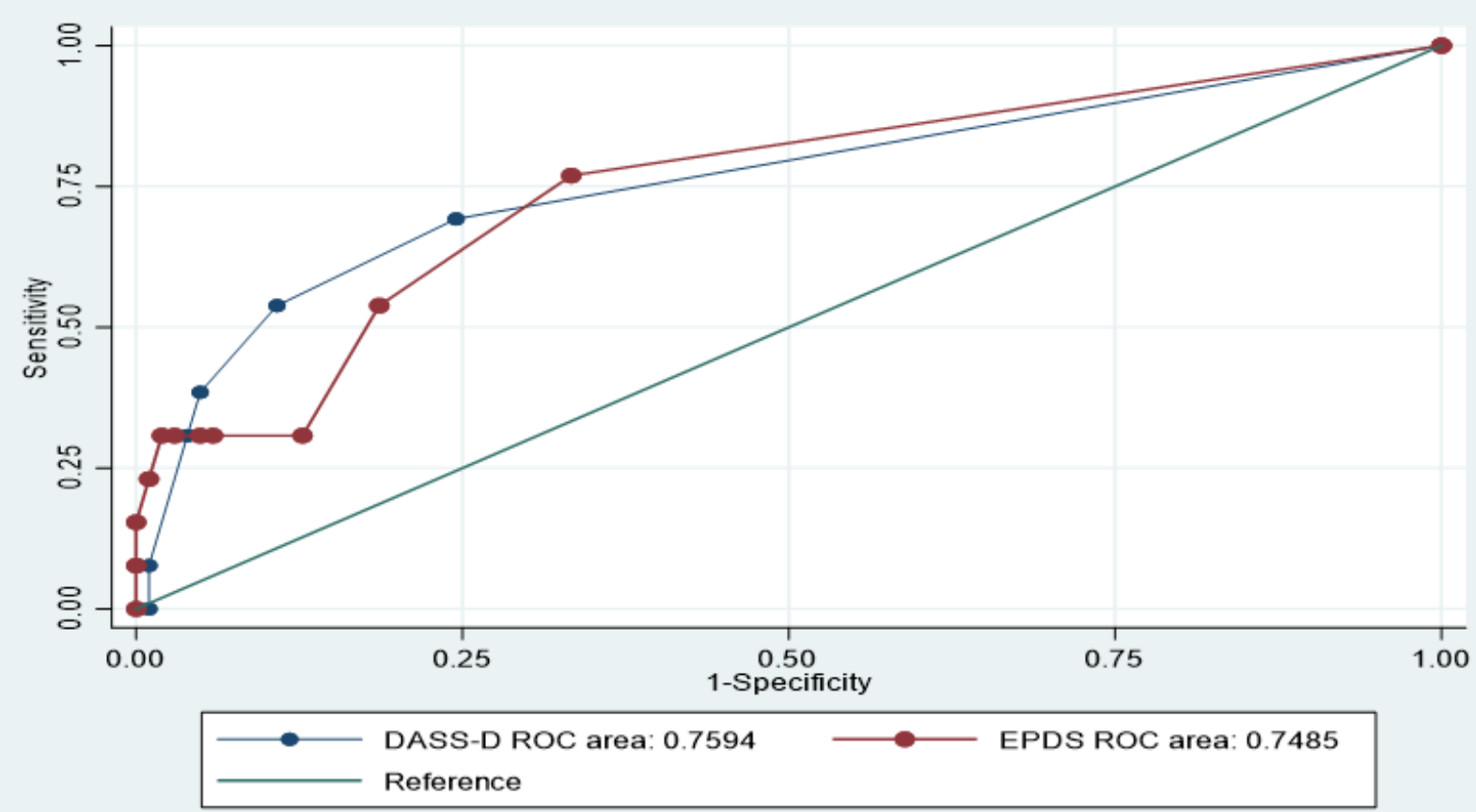

a

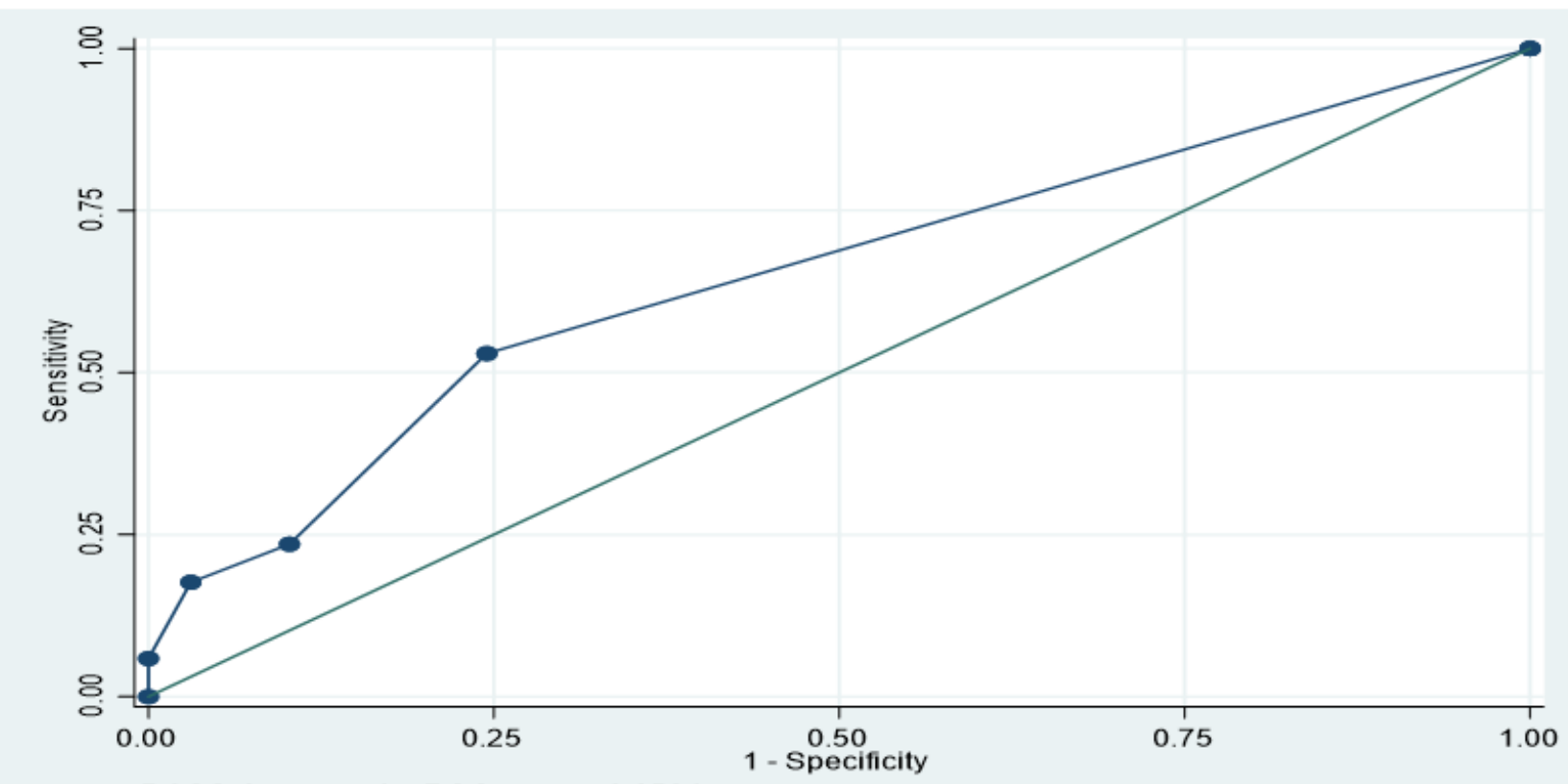

DASS-A area under ROC curve $=0.6504$

b

\section{Figure 1}

$\mathrm{A}$; Comparing predictive ability of DASS-D and EPDS in screening postpartum depression Ho: DASS-D ROC area $=$ EPDS ROC area; chi2 $(1)=0.02$, Prob $>$ chi2 $=0.8844$ B: Predicting ability of DASS-A in screening current generalised anxiety disorder 


\section{Supplementary Files}

This is a list of supplementary files associated with this preprint. Click to download.

- Appendix1DASS21ChichewaVersion.docx 